\title{
Hypertonic Saline Solution for Signal Transmission and Steering in MRI-guided Intravascular Catheterisation
}

\author{
Alberto Caenazzo and Kaspar Althoefer \\ Centre for Advanced Robotics @ Queen Mary (ARQ) \\ Queen Mary University of London, Mile End Road, London, E1 4NS, United Kingdom \\ A.Caenazzo@qmul.ac.uk, K.Al thoefer@qmul.ac.uk
}

\begin{abstract}
Use of traditional low-impedance sensor leads is highly undesirable in intravascular catheters to be used with MRI guidance; thermal safety and quality of imaging are particularly impacted by these components. In this paper, we are showing that hypertonic saline solution, a high-impedance body-like fluid, could be a compatible and effective signal transmission medium when used in MRI-compatible catheters. We also propose a simple type of catheter design that can be steered hydraulically using the same saline solution. Integration of hydraulic steering is not required for MRI-compatibility; however efficient design can bring advantages in terms of structural simplicity and miniaturisation. Manufacturing of proof-of-concept prototypes using 3D printing is underway.
\end{abstract}

Keywords: Surgical robotics, MRI, Soft-robotics, Interventional Electrophysiology

\section{Introduction}

Intravascular catheterization is nowadays a standard technique for many types of minimally invasive diagnostic and surgical procedures. Catheters are typically guided using fluoroscopy - however, this imaging technique uses ionizing radiation and is associated with an increase in risk of cancer and other side effects for patients and attending medical staff. Real-time Magnetic Resonance Imaging (MRI) has been recently developed as a safer, more effective alternative to fluoroscopy; however, practical implementation of MRI is not proving easy. Some intravascular procedures (e.g. interventional electrophysiology studies) require electrical signals to be transmitted outside the body - this is almost universally achieved with thin metal or otherwise low-impedance wires. Unfortunately, such components are particularly undesirable in an MRI environment as they have the potential of heating to dangerous levels; imaging artefacts may also arise, making guidance and positioning of catheters particularly 
difficult [1]. While methods devised to increase MRI-compatibility of "conventional" wire-based systems have been reported in literature [2,3], these same methods are not guaranteed to solve the MRI compatibility issues completely. As a result, MRI-guided intravascular catheterisation is reported to be still largely confined to research environments [3].

\section{Methodology}

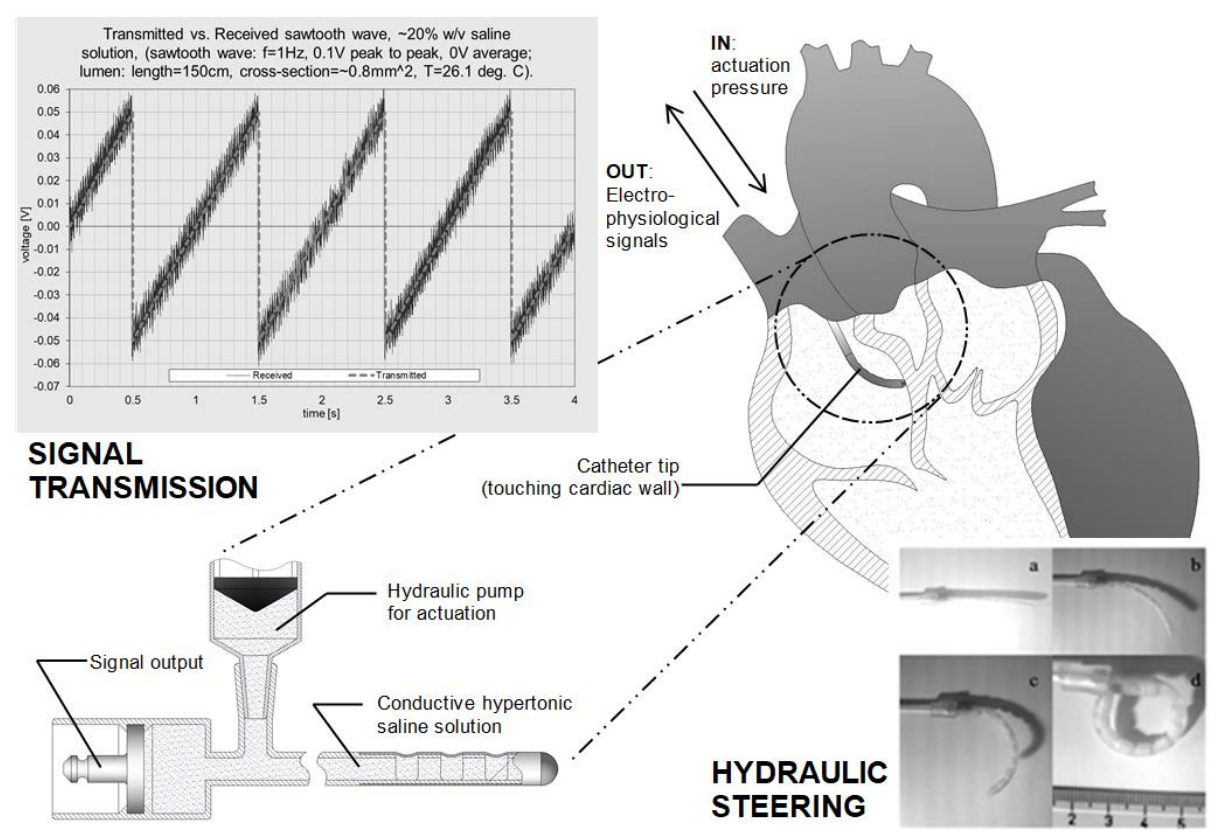

Fig. 1. Our vision. A catheter using the same hypertonic saline solution for signal transmission and steering is used to collect electrophysiological signals from inside the heart.

\subsection{Conduction of electro-physiological signals through saline solution}

Our approach takes inspiration from non-invasive examinations of the electrical signals generated by the heart, e.g. electro-cardiography. With some rough approximation, these signals are collected after passing bodily tissues; these, in turn, can be roughly approximated as normal saline solution ( $0.9 \%$ weight/volume), which is itself a rather poor conductor. Performance of such systems is, however, very well established. Stemming from this, we determined that hypertonic concentrations (i.e. well above normal concentration) offer good signal transmission properties when tested in narrow catheter-like lumens ( $1 \mathrm{~mm}$ internal diameter, $100+\mathrm{cm}$ in length) at similar voltages and frequencies as cardiac electrophysiological signals collected directly. Being similar in composition to human tissues and only marginally more conductive, hypertonic saline solution appears feasible as a MRI-compatible substitute of traditional low-impedance wires. The safety profile of hypertonic saline solution in case of 
accidental spillage into the bloodstream is also very good, with $20 \%$ and $7 \%$ considered as safe concentrations for adult and paediatric patients respectively.

DC electrical properties of saline solutions are well known. Using widely available data and equations [4], we extrapolated the following equation for resistivity vs. concentration and temperature:

$$
\rho_{T^{\circ} \mathrm{C}}=\frac{1}{(16.283 \mathrm{c}+1.0074)[1+0.02(T-25)]}[\mathrm{k} \Omega \cdot \mathrm{cm}]
$$

with $\rho$ resistivity of the solution (in $\mathrm{k} \Omega \cdot \mathrm{cm}$ ) $\mathrm{c}$ weight over volume concentration (in $\mathrm{g} / 100 \mathrm{ml}$ ), $\mathrm{T}$ the temperature of the solution (in degrees Celsius). Recalling Ohm's laws, the resistance of a conductor is directly dependent on its length and resistivity, while it is inversely dependent on its cross section:

$$
R=\rho \frac{l}{A}[\mathrm{k} \Omega]
$$

where R is the resistance (in $\mathrm{k} \Omega$ ), $\rho$ resistivity of the solution (in $\mathrm{k} \Omega \cdot \mathrm{cm}$ ), 1 and $\mathrm{A}$ respectively the length (in $\mathrm{cm}$ ) and the cross section $\left(\right.$ in $\mathrm{cm}^{2}$ ) of the conductor.

Assuming catheter-like dimensions of $1=150 \mathrm{~cm}$ and $\mathrm{A}=0.008 \mathrm{~cm}^{2}$ (approximately equivalent to a lumen of $1 \mathrm{~mm}$ in diameter), we used (1) and (2) to compute the resistance values for different temperatures and concentrations of $\mathrm{NaCl}$ (Fig. 2). Values shown in Fig. 2 are broadly in the same order of magnitude of the impedance normally encountered at interface between body and electrodes in non-invasive studies [5], albeit exclusively calculated here in terms of DC behaviour.

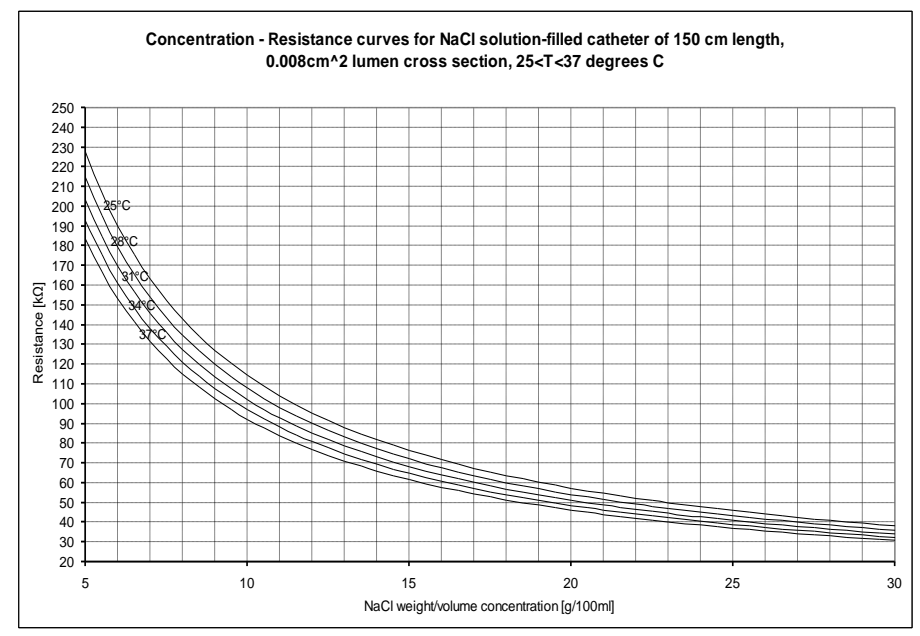

Fig. 2. Calculated DC concentration vs. resistance curves for saline solution in a catheter-like structure at various service temperatures. 
We conducted several experiments by sending and receiving electric signals with frequency and amplitude comparable to what encountered inside the heart (frequency $=1 \mathrm{~Hz}$, amplitude $=0.1 \mathrm{~V}$ peak to peak) through a catheter-like lumen. The equivalent circuit schematic of the test rigs we used is shown in Fig. 3.

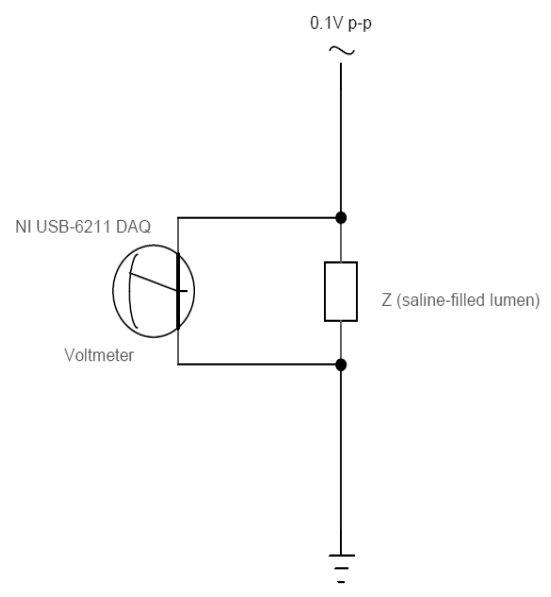

Fig. 3. Equivalent circuit for the experimental test-rigs.

The experimental results proved the feasibility of the approach; a clear example is shown in the chart on top left corner of Fig. 1, replicated for clarity in Fig. 4 below. In this chart, the signal received through the catheter-like lumen follows very closely the transmitted signal (net of the $50 \mathrm{~Hz}$ mains noise captured by the unshielded test-rig).

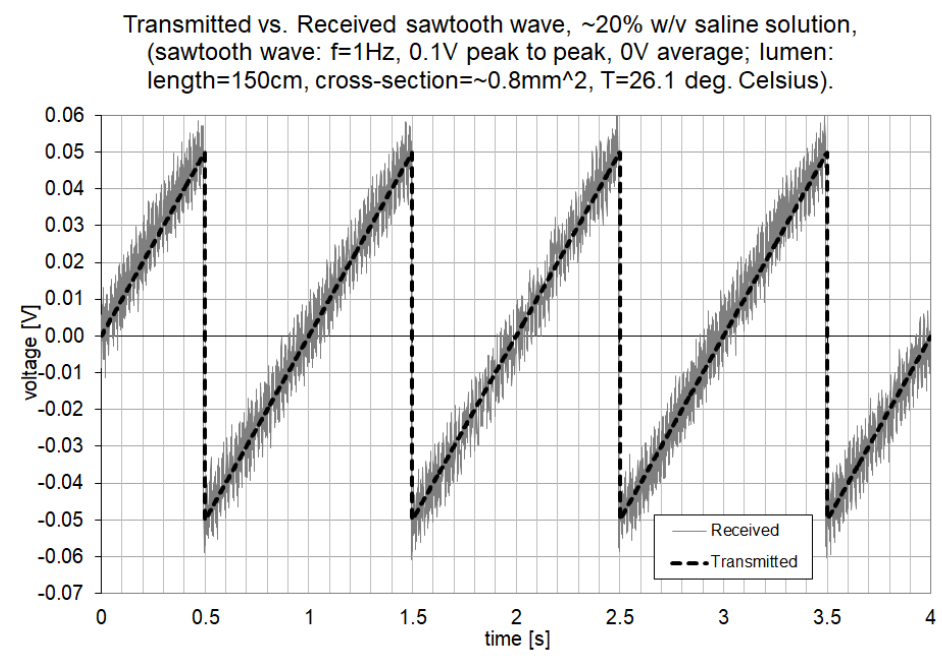

Fig. 4. Experimental results of signal transmission through a catheter-like geometry filled with saline solution. 


\subsection{Integration of hydraulic catheter steering}

We also investigated the integration of hydraulic steering using the same conductive fluid. MRI-compatible steering mechanisms can be devised without the need of an hydraulic system, e.g. using tendons or push-rods made of MRI-compatible polymers [6]. However, it can make sense to re-use/re-purpose components that are available by design in the body of the catheter - this could help in designing a smaller and/or simpler structure (reducing stress on blood vessels and being easier/cheaper to manufacture, respectively), or could even be used as part of an hybrid hydraulic-tendon system with improved mechanical characteristics [7]. Our proof-of-concept design uses a very simple 1-DOF mechanism (Fig. 5) that is steered by removing a small volume of fluid, and re-straightened by pumping in back the same volume (Fig. 6) [8,9].

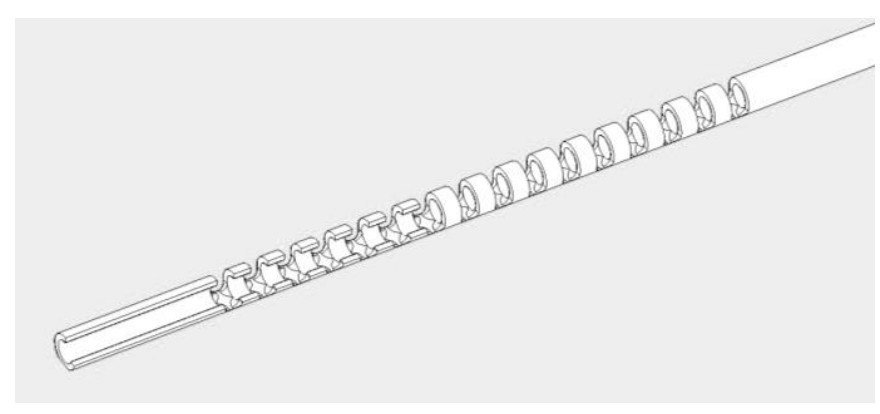

Fig. 5. Rendering of the simple hydraulic steerable catheter structure we are using as proof-ofconcept. Note that the LHS of the structure is sectioned to show the internal lumen. In order for the catheter to work as intended, a thin silicone "skin" needs to cover the catheter structure. This is not shown in this rendering, but is visible in the real-life prototype pictures (Fig. 1 and 8-9) and the schematic in Fig. 6.

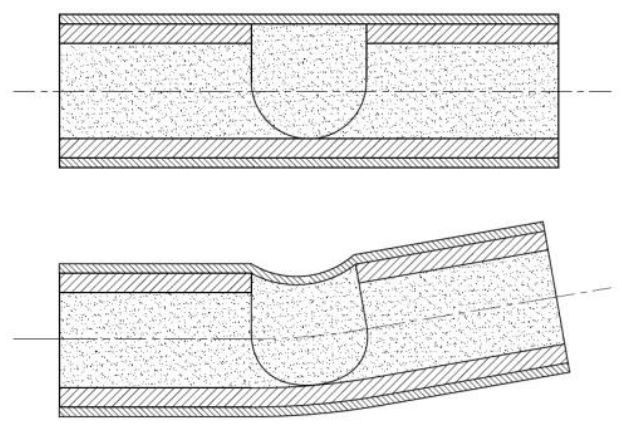

Fig. 6. Schematic showing the basics of the prototype hydraulic actuation. The top drawing shows a section of the catheter at rest - note the "skin" covering the entire catheter structure. The bottom configuration shows the effect of displacing some fluid from the catheter: the skin collapses over the groove, in turn causing the entire structure to bend. While not in scope for this paper or the prototypes shown here, it is noteworthy that rigorous modelling has been developed to determine the relationship between bending radius and fluid displacement. This, in turn, can allow for development of robotic control systems and trajectory-planning algorithms. 
Variances in the conductive cross-section (thus in resistivity) in the catheter tip are still very limited when actuating the steering mechanism, thus not affecting the overall signal transmission properties of the whole catheter. After some initial successes with simple handcrafted samples [9], we are currently in the advanced manufacturing stages of a functional prototype, while at the same time testing designs, materials and assembly techniques that can be useful in future production. Use of 3D printing has enabled to significantly reduce the time and costs involved to test ideas, with results that are more than satisfactory considering the minuscule size of the components being printed and the consumer-grade technology employed so far (e.g. Fig. 7, 8, 9).

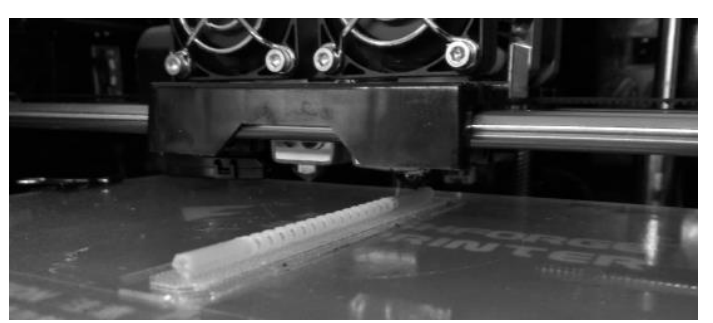

Fig. 7. Catheter structure being printed. We used a fairly standard, consumer-grade Fused Filament Fabrication printer with a variety of materials.

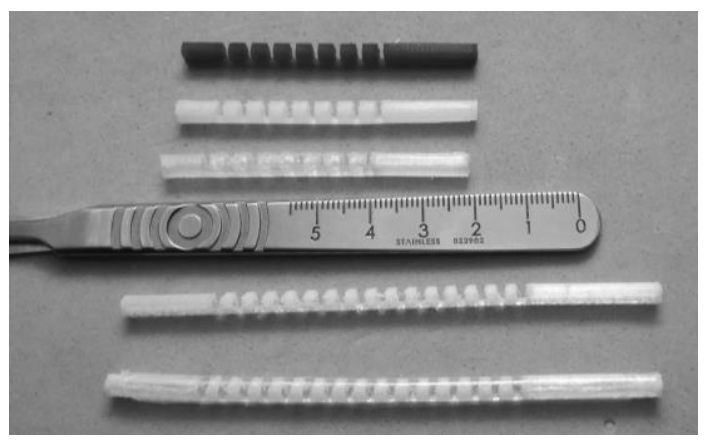

Fig. 8. Examples of catheter structures printed with different materials. From top to bottom: PLA (non-steerable), Nylon, Flexible TPU, PETG, PETG with silicone skin added. Note the realistic scale of the structures (3-4 mm outer diameter).

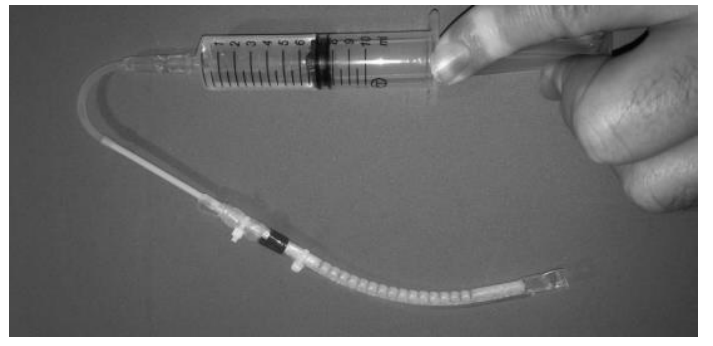

Fig. 9. Assembled catheter prototype being steered hydraulically. This is a PETG structure with a silicone skin of the same type as shown in Fig. 8. 


\section{Conclusions}

Stemming from our positive experimental results, we can state that use of hypertonic saline solution can indeed be evaluated as a viable alternative to traditional metal/lowimpedance wires for signal transmission in MRI-compatible intravascular catheters. We also examined the integration between signal transmission and hydraulic steering, as exemplified by our realistic-sized 3D-printed prototypes. More work is required to demonstrate the effectiveness of our approach in-vivo and in the actual MRI setting.

\section{References}

1. M. K. Koning, S. Weiss, C. J. G. Bakker, L. W. Bartels, W. P. T. M. Mali, Catheters and guidewires in interventional MRI: problems and solutions, 2001, Medica Mundi 45/1, 3139.

2. P. Bhagirat, M. van der Graaf, R. Karim, K. Rhode, C. Piorkowski, R. Razavi, J. Schwitter, M. Goette, Interventional Cardiac Magnetic Resonance Imaging in Electrophysiology, 2014.

3. H. Chubb, S. E. Williams, J. Whitaker, J. L. Harrison, R. Razavi, M. O’Neill, Cardiac Electrophysiology Under MRI Guidance: An Emerging Technology, Arrhythmia \& Electrophysiology Review 2017;6(2):85-3

4. Radiometer Analytical, Conductivity, Theory and Practice. Villerurbanne, France, 2004.

5. S. Liu, J. Kruse, Biopotential Electrode Sensors in ECG/EEG/EMG Systems. Analog Devices Inc., 2008.

6. A. Ataollahi, R. Karim, A. S. Fallah, K. Rhode, R. Razavi, L. D. Seneviratne, T. Schaeffter, K. Althoefer, Three-Degree-of-Freedom MR-Compatible Multisegment Cardiac Catheter Steering Mechanism, IEEE Transactions on Biomedical Engineering 63 (11), 2425-2435, 2013

7. A. Stilli, H. Wurdemann, K. Althoefer, Shrinkable, stiffness-controllable soft manipulator based on a bio-inspired antagonistic actuation principle, 2014 IEEE/RSJ International Conference on Intelligent Robots and Systems (IROS 2014)

8. Haga Y. et al., Small diameter hydraulic active bending catheter using laser processed super elastic alloy and silicone rubber tube, Proceedings of the 3rd Annual International IEEE EMBS Special Topic Conference on Microtechnologies in Medicine and Biology 2005;245-248

9. A. Caenazzo, H. Liu, K. Althoefer, Integrated Signal Transmission And Hydraulic Steering Using Hypertonic Saline Solution In MRI-Compatible Intravascular Catheters, Abstract book of the 24th Congress of EAES, 2016 